\title{
Thermally induced evolution of phase transformations in gas hydrate sediment
}

\author{
ZHANG XuHui $^{1 *}$, LU XiaoBing $^{1}$, LI QingPing ${ }^{2}$ \& YAO HaiYuan ${ }^{2}$ \\ ${ }^{1}$ Key Laboratory for Hydrodynamics and Ocean Engineering, Institute of Mechanics, Chinese Academy of Sciences, Beijing 100190, China; \\ ${ }^{2}$ Research Centre, China Ocean Oil Co., Beijing 100027, China
}

Received October 23, 2009; accepted May 10, 2010; published online July 9, 2010

\begin{abstract}
Thermally induced evolution of phase transformations is a basic physical-chemical process in the dissociation of gas hydrate in sediment (GHS). Heat transfer leads to the weakening of the bed soil and the simultaneous establishment of a time varying stress field accompanied by seepage of fluids and deformation of the soil. As a consequence, ground failure could occur causing engineering damage or/and environmental disaster. This paper presents a simplified analysis of the thermal process by assuming that thermal conduction can be decoupled from the flow and deformation process. It is further assumed that phase transformations take place instantaneously. Analytical and numerical results are given for several examples of simplified geometry. Experiments using Tetra-hydro-furan hydrate sediments were carried out in our laboratory to check the theory. By comparison, the theoretical, numerical and experimental results on the evolution of dissociation fronts and temperature in the sediment are found to be in good agreement.
\end{abstract}

gas hydrate, dissociation front, phase transformation, thermal conduction

PACS: 07.10.Pz, 44.05.+e, 47.56.+r, 62.20.Mk, 91.50.Hc

Natural gas hydrate (NGH) is a crystalline solid composed mainly of methane gas and water molecules, and is stabilized at high pressure and low temperature [1,2]. NGH is extensively distributed in sediments of oceans, continental margins, permafrost zones and deep lakes $[3,4]$. When the phase equilibrium is destabilized, NGH will dissociate into gas and water. Generally, $1 \mathrm{~m}^{3}$ of NGH may release $164 \mathrm{~m}^{3}$ methane gas and $0.8 \mathrm{~m}^{3}$ of water at $1 \mathrm{~atm}$ at normal temperature. Then a large excess pore pressure will result in a strength decrease of the sediment if the released gas diffuses slowly [5,6].

During exploitation of gas hydrate or gas and oil in the deep sea, high-temperature pipes will pass through GHS and make the temperature of GHS increase. Accordingly, NGH in sediment will dissociate and the GHS and cap-rock become unstable. The dissociation zone extends with time and

*Corresponding author (email: zhangxuhui@imech.ac.cn) the strength of the sediment reduces due to the increase of excess pore pressure and the loss of cementation. This may lead to cracks in the base of the structures and overturn of platforms, pipe rupture, and even blowout [7-10].

Thermal dissociation of the hydrate in GHS is related with three temporal characteristic parameters: thermal conduction time $t_{\mathrm{c}}^{*}=\rho C l^{2} / K$, seepage time $t_{p}^{*}=\mu_{g} l^{2} / k_{g} p_{e}$, and time of elastic wave propagation $t_{\mathrm{e}}^{*}=l / \sqrt{E / \rho}$. Their ratio is about $10^{9}: 10^{5}: 1$. That means the problem involves three independent processes, i.e. thermal conduction, seepage, and elastic wave propagation. Thus we first analyze the thermal conduction involving phase transition, and then the pressure distribution in sediment, and finally the failure of sediment due to redistribution of stresses.

Tetra-hydro-furan (THF) is liquid at $0.1 \mathrm{MPa}$, and can dissolve in water with any proportion. It can form a type- II hydrate with water at $4.4^{\circ} \mathrm{C}[11,12]$. The enthalpy of 
THF hydrate dissociation is $270 \mathrm{~kJ} / \mathrm{kg}$ at $4.4^{\circ} \mathrm{C}$ [13]. Many researches have been carried out to investigate the thermal properties of THF hydrate and the induction time with different proportions in solution [14-17]. By comparison, THF hydrate has similar thermal properties with methane hydrate, i.e. $1<\left(\frac{\Delta H}{C_{h}}\right)_{\mathrm{MH}} /\left(\frac{\Delta H}{C_{h}}\right)_{\mathrm{TFH}}<10$ and $K_{\mathrm{MH}} / K_{\mathrm{TFH}} \approx 1$, and its synthesis is relatively simple, economical and safe.

This paper is devoted to presenting a thermal conduction model involving phase transformations. Analytical and numerical approaches for several simplified examples of geometry are made, then experiments using THF hydrate sediments are carried out to check the theory.

\section{Assumption of phase transformation front}

Two kinds of models are presented to describe hydrate phase transition. One is regarded as the front-melting process [18], i.e. hydrates dissociate instantly once the phase equilibrium condition is not satisfied. Conservation of energy and mass is not fully considered in this case. The other one is taken as a kinetic process in which parameters are difficult to obtain [19]. In this paper, phase transformations containing hydrate transformation are assumed to be front-melting processes but energy and mass conservation are considered at the fronts. Four zones are formed in the sediment: un-dissociated zone, hydrate dissociation zone, gasification zone, and water vaporization zone. Vaporization here indicates water boiling.

The formation mechanism can be described as follows: firstly, the temperature of the sediment surrounding the thermal source increases, leading hydrates to dissociate into water and liquid gas when the phase equilibrium temperature is reached, then the hydrate dissociation zone and the un-dissociated zone are formed and divided by the dissociation front; secondly, liquid THF is gasified when the gasification temperature is reached, then the hydrate dissociation zone and the gasification zone are formed and divided by the gasification front; Finally, water is transformed into vapor when the boiling temperature is reached, then the gasification zone and water vaporization zone are formed and divided by the vaporization front. These three fronts develop with time. the temperature distribution, phase transition front, and formation of four zones in the hydrate sediment are shown schematically in Figure 1, in which each zone is characteristic of a mixture: (1) water vaporization zone: sediment skeleton, water vapor, gas; (2) gasification zone: gas, water and sediment skeleton; (3) hydrate dissociation zone: liquid gas, water and sediment skeleton; (4) un-dissociated zone: hydrate and sediment skeleton. In this figure, (1), (2) and (3) represent the water vaporization front, the gasification front and the dissociation front, respectively.

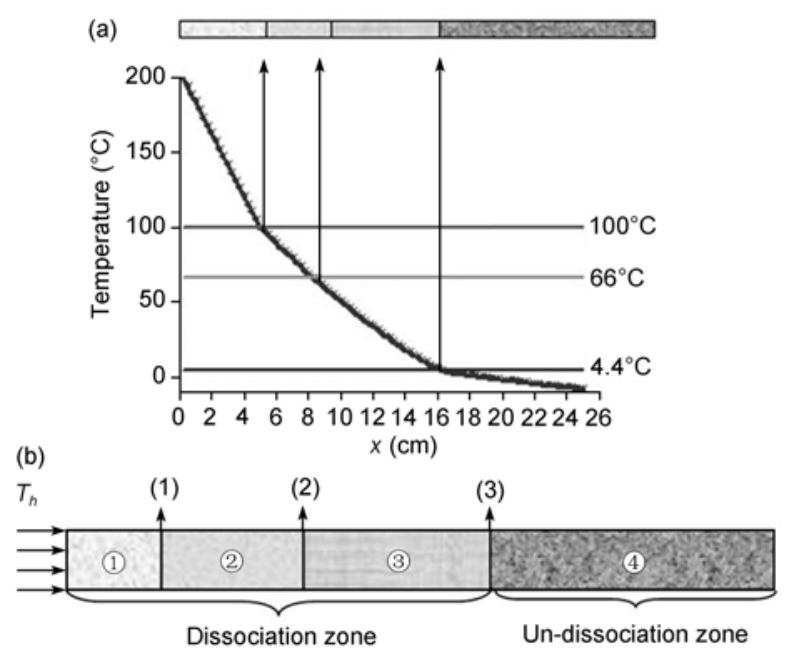

Figure 1 Temperature distribution and division of four zones. (a) Temperature distribution; (b) four zones and phase transformation fronts.

\section{Mathematical model}

In order to formulate the thermal evolution, the following assumptions are adopted: (1) Thermodynamic parameters in every mixture zone are taken as the average of each phase. (2) The enthalpies of water, THF and the hydrate are taken as constants. Based on the mixture theory, a one-dimensional equation of thermal dissociation of the hydrate is obtained:

$$
\begin{aligned}
C \rho \frac{\partial T}{\partial t}= & K \frac{\partial^{2} T}{\partial x^{2}}+\rho_{h} \Delta H_{h \rightarrow w+f} \frac{\partial \varepsilon_{h}}{\partial t}+\rho_{f} \Delta H_{f \rightarrow f g} \frac{\partial \varepsilon_{f}}{\partial t} \\
& +\rho_{w} \Delta H_{w \rightarrow w g} \frac{\partial \varepsilon_{w}}{\partial t} .
\end{aligned}
$$

Here, the thermodynamic parameters in each zone are as follows:

$$
\begin{aligned}
\rho C= & \varepsilon_{w} \rho_{w} C_{w}+\varepsilon_{w g} \rho_{w g} C_{w}+\varepsilon_{f} \rho_{f} C_{f} \\
& +\varepsilon_{f g} \rho_{f g} C_{f g}+\varepsilon_{r} \rho_{r} C_{r}+\varepsilon_{h} \rho_{h} C_{h}, \\
K= & \varepsilon_{w} K_{w}+\varepsilon_{w g} K_{w g}+\varepsilon_{f} K_{f}+\varepsilon_{f g} K_{f g} \\
& +\varepsilon_{r} K_{r}+\varepsilon_{h} K_{h} .
\end{aligned}
$$

in which $f, h, r, w g, f g$ indicate THF, water, THF hydrate, sediment skeleton, water vapor and THF gas respectively, $\rho$, $C$ and $K$ indicate mean density, specific heat and coefficient of heat conduction respectively, and $\varepsilon$ is the volume fraction.

Based on the phase transformation assumption, two conclusions are obtained: (1) phase transformation occurs once both the temperature and the provided energy satisfy the required conditions, and so the following equations are satisfied: $\quad \varepsilon_{h}=H\left(x-s_{h}(t)\right), \quad \varepsilon_{f}=H\left(x-s_{f}(t)\right), \quad \varepsilon_{w}=H(x-$ $\left.s_{w}(t)\right)$; (2) temperature is continuous and equal to that at the phase equilibrium point at the phase transformation front, i.e. 
$T\left(s_{i}\right)=T_{D i}$.

Substitute assumption (1) into eq. (1) and integrate two sides of the phase transformation front, so we get:

$$
\left.K \frac{\partial T}{\partial x}\right|_{s_{i}+}-K \frac{\partial T}{\partial x_{s_{i}-}}=\rho_{i} \Delta H \varepsilon_{i} \frac{\mathrm{d} s_{i}}{\mathrm{~d} t} .
$$

Eq. (2) shows the joined condition of temperature gradient at the phase transformation front and indicates the conservation of energy. The index $i$ represents $f, w, h$, respectively.

Then the model is as follows:

Governing equation:

$$
\rho C \frac{\partial T}{\partial t}=K \frac{\partial^{2} T}{\partial x^{2}}
$$

Boundary condition:

$$
x=0, T=T_{H} \text { and } x=l, T=T_{0} .
$$

Initial condition:

$$
t=0, T=T_{0} .
$$

Joined condition at phase transformation fronts:

$$
T\left(s_{i}(t)\right)=T_{D i}
$$

and

$$
\left.K \frac{\partial T}{\partial x}\right|_{s_{i}+}-\left.K \frac{\partial T}{\partial x}\right|_{s_{i}-}=\rho_{i} \Delta H \varepsilon_{i} \frac{\mathrm{d} s_{i}}{\mathrm{~d} t} .
$$

\section{Numerical simulation}

Numerical simulation is utilized here to analyze the development of temperature and temperature gradient based on the Crank-Nicolson difference method. The parameters are shown in the appendix. The density of gas at the maximum pressure $0.1 \mathrm{MPa}$ in experiments is adopted. The specific heat of gas is determined by $C=3 R / M$. The heat conduction coefficient is assumed to be the same as that of water vapor though it is changeable with temperature. Analysis shows that the approximate selection of gas thermodynamic parameters is insensitive to calculated results.

Figure 2(a) shows the evolution of temperature distribution when the thermal source is $200^{\circ} \mathrm{C}$. It is shown that the temperature difference near the thermal source decreases with time, which means there is a decrease of the rate of input energy. The temperature distribution becomes stable until the input and output energies are equal. Figure 2(b) shows the evolution of temperature gradients. Apparent discontinuities appear at the phase transformation fronts. The results verify that the temperature is continuous and the temperature gradient is discontinuous at phase transformation fronts.

\section{Self-similarity solution}

The controlling parameters of thermal conduction are as follows: temperature of thermal source $T_{h}$, initial temperature of sediment $T_{i}$, specific heat $C$, thermal conduction coefficient $K$ and density $\rho$. Temperature evolution can be written in the dimensionless form: $\frac{T-T_{0}}{T_{h}-T_{0}}=f\left(\frac{\rho C x^{2}}{K t}\right)$. Let $\theta=$ $\frac{T-T_{0}}{T_{h}-T_{0}}, \quad \eta=\frac{\rho C x^{2}}{K t}$, then eq. (3) can be rewritten as:

$$
4 \frac{\mathrm{d}^{2} f}{\mathrm{~d} \eta^{2}}+\left(1+\frac{2}{\eta}\right) \frac{\mathrm{d} f}{\mathrm{~d} \eta}=0
$$

Obviously, the ordinary differential equation eq. (7) is easier to solve than the partial differential equation eq. (3), and its general self-similar solution is:

$$
\begin{aligned}
f & =A \cdot\left(\frac{2}{\sqrt{\pi}} \int_{0}^{\frac{x}{2 \sqrt{\beta t}}} \exp \left(-\xi^{2}\right) \mathrm{d} \xi\right)+B \\
& =A \cdot \operatorname{erf}\left(\frac{x}{2 \sqrt{\beta t}}\right)+B,
\end{aligned}
$$

in which $\beta=\frac{\rho C}{K}$.

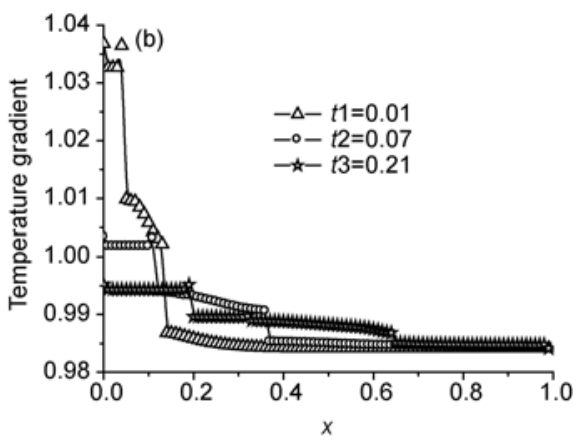

Figure 2 Distribution of temperature and temperature gradient under constant boundary temperature. (a) Evolution of temperature; (b) evolution of temperature gradient. 
Self-similar solutions in each zone can be written as $\theta_{i}=A_{i} \cdot \operatorname{erf}\left(\frac{x}{2 \sqrt{\beta_{i} t}}\right)+B_{i}$, in which $i$ indicates the zone number $1,2,3$, and $4 . A_{i}$, and $B_{i}$ can be solved according to respective boundary conditions.

In zone 1: $A_{1}$ and $B_{1}$ are obtained according to $\left.T\right|_{x=0}=T_{h}$ and $x=s_{1}(t),\left.\quad T\right|_{x=s_{1}(t)}=T_{e 1}$ as:

$$
A_{1}=\frac{T_{e 1}-T_{h}}{\operatorname{erf}\left(\lambda_{1}\right)} \text { and } B_{1}=T_{h} \text {. }
$$

In zone 2: According to $\left.T\right|_{x=s_{2}(t)}=T_{e 2}$ and $\left.T\right|_{x=s_{1}(t)}=T_{e 1}, A_{2}$ and $B_{2}$ are obtained as:

$$
\begin{aligned}
A_{2}= & \frac{T_{e 2}-T_{e 1}}{\operatorname{erf}\left(\lambda_{2}\right)-\operatorname{erf}\left(\lambda_{1} \sqrt{\frac{\beta_{1}}{\beta_{2}}}\right)}, \\
B_{2}= & T_{e 2}-\frac{T_{e 2}-T_{e 1}}{\operatorname{erf}\left(\lambda_{2}\right)-\operatorname{erf}\left(\lambda_{1} \sqrt{\frac{\beta_{1}}{\beta_{2}}}\right)} \cdot \operatorname{erf}\left(\lambda_{2}\right) .
\end{aligned}
$$

In zone 3: According to $\left.T\right|_{x=s_{3}(t)}=T_{e 3}$ and $\left.T\right|_{x=s_{2}(t)}=T_{e 2}, A_{3}$ and $B_{3}$ are obtained as:

$$
\begin{aligned}
& A_{3}=\frac{T_{e 3}-T_{e 2}}{\operatorname{erf}\left(\lambda_{3}\right)-\operatorname{erf}\left(\lambda_{2} \sqrt{\frac{\beta_{2}}{\beta_{3}}}\right)}, \\
& B_{3}=T_{e 3}-\frac{T_{e 3}-T_{e 2}}{\operatorname{erf}\left(\lambda_{3}\right)-\operatorname{erf}\left(\lambda_{2} \sqrt{\frac{\beta_{2}}{\beta_{3}}}\right)} \cdot \operatorname{erf}\left(\lambda_{2}\right) .
\end{aligned}
$$

In zone 4: $A_{4}$ and $B_{4}$ are obtained according to $\left.T\right|_{x=l}=T_{0}$ and $\left.T\right|_{x=s_{3}(t)}=T_{e 3}$ :

$$
\begin{aligned}
& A_{4}=\frac{T_{0}-T_{e 3}}{1-\operatorname{erf}\left(\lambda_{3} \sqrt{\frac{\beta_{3}}{\beta_{4}}}\right)}, \\
& B_{4}=T_{0}-\frac{T_{0}-T_{e 3}}{1-\operatorname{erf}\left(\lambda \sqrt{\frac{\beta_{3}}{\beta_{4}}}\right)} .
\end{aligned}
$$

Substitute eqs. (9)-(12) into the joined conditions of temperature gradient, so we obtain:

$$
K_{2} \frac{T_{e 2}-T_{e 1}}{\operatorname{erf}\left(\lambda_{2}\right)-\operatorname{erf}\left(\lambda_{1} \sqrt{\frac{\beta_{1}}{\beta_{2}}}\right)} \sqrt{\frac{\beta_{1}}{\beta_{2}}} \exp \left(-\frac{\beta_{1}}{\beta_{2}} \lambda_{1}^{2}\right)
$$

$$
\begin{aligned}
& -K_{1} \frac{T_{e 1}-T_{h}}{\operatorname{erf}\left(\lambda_{1}\right)} \exp \left(-\lambda_{1}^{2}\right)=\rho_{w} \Delta H_{w \rightarrow g} \varepsilon_{w} \lambda_{1} \beta_{1} \sqrt{\pi}, \\
& K_{3} \frac{T_{e 3}-T_{e 2}}{\operatorname{erf}\left(\lambda_{3}\right)-\operatorname{erf}\left(\lambda_{2} \sqrt{\frac{\beta_{2}}{\beta_{3}}}\right)} \sqrt{\frac{\beta_{2}}{\beta_{3}}} \exp \left(-\frac{\beta_{2}}{\beta_{3}} \lambda_{2}^{2}\right) \\
& -K_{2} \frac{T_{e 2}-T_{e 1}}{\operatorname{erf}\left(\lambda_{2}\right)-\operatorname{erf}\left(\lambda_{1} \sqrt{\frac{\beta_{1}}{\beta_{2}}}\right)} \exp \left(-\lambda_{2}^{2}\right) \\
& =\rho_{f} \Delta H_{f \rightarrow g} \varepsilon_{f} \lambda_{2} \beta_{2} \sqrt{\pi} \text {, }
\end{aligned}
$$

$$
\begin{aligned}
& K_{4} \frac{T_{0}-T_{e 3}}{1-\operatorname{erf}\left(\lambda_{3} \sqrt{\frac{\beta_{3}}{\beta_{4}}}\right)} \sqrt{\frac{\beta_{3}}{\beta_{4}}} \exp \left(-\frac{\beta_{3}}{\beta_{4}} \lambda_{3}^{2}\right) \\
& -K_{3} \frac{T_{e 3}-T_{e 2}}{\operatorname{erf}\left(\lambda_{3}\right)-\operatorname{erf}\left(\lambda_{2} \sqrt{\frac{\beta_{2}}{\beta_{3}}}\right)} \exp \left(-\lambda_{3}^{2}\right) \\
& =\rho_{h} \Delta H_{h \rightarrow f} \varepsilon_{h} \lambda_{3} \beta_{3} \sqrt{\pi} \text {. }
\end{aligned}
$$

Thus $\lambda_{1}, \lambda_{2}$ and $\lambda_{3}$ can be determined and the whole solution of the problem is obtained.

\section{Model experiments}

The model box has a size of: length $\times$ width $\times$ height $=50 \mathrm{~cm}$ $\times 10 \mathrm{~cm} \times 30 \mathrm{~cm}$. A temperature controller and an immersion heater are touched closely to form a thermal source which can provide a stable temperature in the range of $-30-300^{\circ} \mathrm{C}$. A stopwatch is adopted to record the time. The development of a dissociation front can be regarded as one dimensional once the front arrives at the sidewall. Before that, it can be thought of as axially-symmetric. The experimental procedures are as follows: (1) put fine sand with a dry density of $1.6 \mathrm{~g} / \mathrm{cm}^{3}$ into the box as the skeleton of sediments and insert a temperature controller and an immersion heater vertically in the middle of the box; (2) saturate the sediment with a $19 \%$ (mass fraction) THF solution; (3) put the box into a refrigerator at $-8^{\circ} \mathrm{C}$ for about $3 \mathrm{~d}$. After the formation of hydrate sediment, the temperature is increased to dissociate the hydrate, and the phase transition front of the THF hydrate with time is recorded. The experiment is stopped when the dissociation fronts do not expand anymore [20]. The layout of the experiment is shown in Figure 3.

A hydrate dissociation front is apparently observed in the experiments. Comparison of the experimental, theoretical and numerical results, with the same parameters, on the evolution of the hydrate dissociation front is shown in Figure 4. It is shown that the results are in good agreement. 


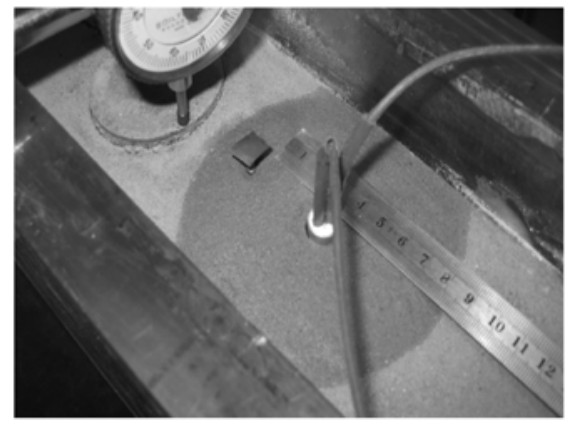

Figure 3 Layout of the experiment.

\section{Conclusions}

Evolution of the hydrate dissociation zone in GHS is im- portant for the evaluation of hazards induced by hydrate dissociation. A new model is proposed to describe the thermal conduction considering the phase transformations. Selfsimilar solutions are obtained. Numerical simulation shows that the temperature is continuous and the temperature gradient is discontinuous at the phase transformation front. Model experiments using THF hydrate sediment are carried out. Results from experiments, theoretical analysis and numerical simulation are in good agreement. However, the developments of gas pressure and gas seepage in sediment were ignored in the present model. Only a hydrate dissociation front is measured experimentally. The model will be improved in future studies. The seepage of released gas and water and the redistribution of pore pressure and stress in sediment also require serious study in the future.
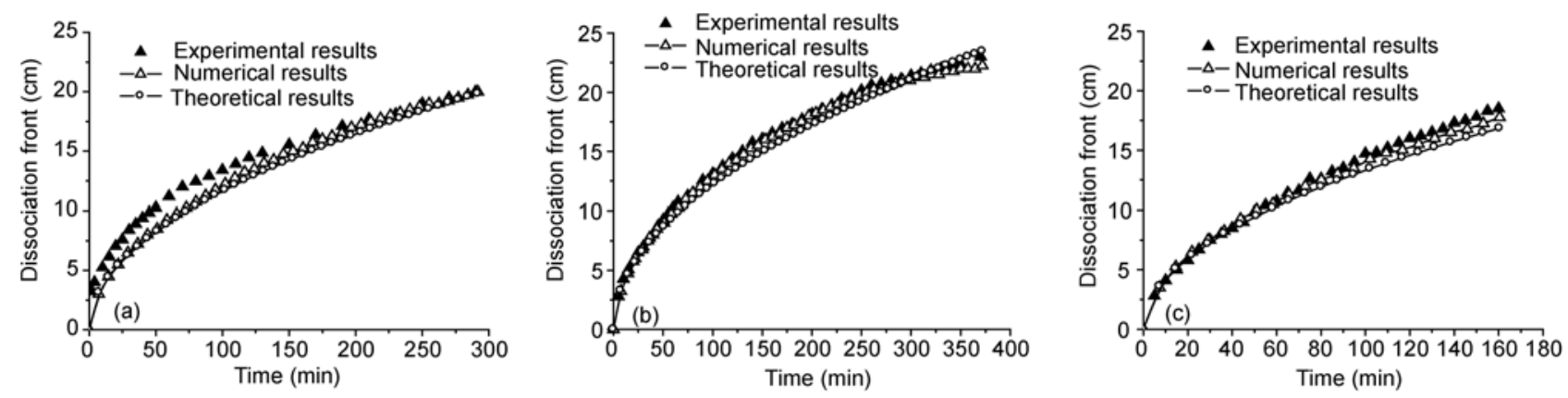

Figure 4 comparison of experimental, simulated and theoretical results. (a) Thermal temperature $150^{\circ} \mathrm{C}$; (b) thermal temperature $200^{\circ} \mathrm{C}$; (c) thermal temperature $300^{\circ} \mathrm{C}$.

\section{Appendix}

Table for basic parameters ${ }^{\text {a) }}$

\begin{tabular}{|c|c|c|c|c|c|}
\hline Label & Parameters & Value & Label & Parameters & Value \\
\hline 1 & Initial temperature & $T_{0}=265.15 \mathrm{~K}$ & 18 & Specific heat of water & $C_{w}=4211 \mathrm{~J} \mathrm{~kg}^{-1} \mathrm{~K}^{-1}$ \\
\hline 2 & Density of sand & $\rho_{r}=2500 \mathrm{~kg} \mathrm{~m}^{-3}$ & 19 & Specific heat of THF gas & $C_{f g}=346 \mathrm{~J} \mathrm{~kg}^{-1} \mathrm{~K}^{-1}$ \\
\hline 3 & Volume fraction of sediment skeleton & $\varepsilon_{r}=0.6$ & 20 & Specific heat of sand skeleton & $C_{r}=840 \mathrm{~J} \mathrm{~kg}^{-1} \mathrm{~K}^{-1}$ \\
\hline 4 & Volume fraction of hydrate & $\varepsilon_{h}=0.4$ & 21 & Specific heat of THF & $C_{f}=4211 \mathrm{~J} \mathrm{~kg}^{-1} \mathrm{~K}^{-1}$ \\
\hline 5 & Length of experimental zone & $l=0.25 \mathrm{~m}$ & 22 & $\begin{array}{l}\text { Thermal conduction coefficient of water } \\
\text { vapor }\end{array}$ & $K_{w g}=0.03 \mathrm{~W} \mathrm{~m}^{-1} \mathrm{~K}^{-1}$ \\
\hline 6 & Density of THF hydrate & $P_{h}=997 \mathrm{~kg} \mathrm{~m}^{-3}$ & 23 & $\begin{array}{l}\text { Thermal conduction coefficient of THF } \\
\text { gas }\end{array}$ & $K_{f g}=0.03 \mathrm{~W} \mathrm{~m}^{-1} \mathrm{~K}^{-1}$ \\
\hline 7 & Thermal conduction coefficient of THF hydrate & $K_{h}=0.46 \mathrm{~W} \mathrm{~m}^{-1} \mathrm{~K}^{-1}$ & 24 & Thermal conduction coefficient of water & $K_{w}=0.56 \mathrm{~W} \mathrm{~m}^{-1} \mathrm{~K}^{-1}$ \\
\hline 8 & Enthalpy of THF hydrate dissociation & $\Delta H_{h-f}=2.7 \times 10^{5} \mathrm{~J} \mathrm{~kg}^{-1}$ & 25 & $\begin{array}{l}\text { Thermal conduction coefficient of sand } \\
\text { skeleton }\end{array}$ & $K_{r}=3 \mathrm{~W} \mathrm{~m}^{-1} \mathrm{~K}^{-1}$ \\
\hline 9 & Phase equilibrium temperature of THF hydrate & $T_{h D}=277.15 \mathrm{~K}$ & 26 & Thermal conduction coefficient of THF & $K_{f}=0.12 \mathrm{~W} \mathrm{~m}^{-1} \mathrm{~K}^{-1}$ \\
\hline 10 & Specific heat of THF hydrate & $C_{h}=2123 \mathrm{~J} \mathrm{~kg}^{-1} \mathrm{~K}^{-1}$ & 27 & Points of difference grid & $N=100$ \\
\hline 11 & Enthalpy of THF gasification & $\Delta H_{f \rightarrow f g}=4.1 \times 10^{5} \mathrm{~J} \mathrm{~kg}^{-1}$ & 28 & Thermal diffusion coefficient & $K / \rho C=10^{-7} \mathrm{~m} \mathrm{~s}^{-1}$ \\
\hline 12 & Density of THF & $\rho_{f}=890 \mathrm{~kg} \mathrm{~m}^{-3}$ & 29 & Gas cohesion coefficient & $\mu_{g}=10^{-5} \mathrm{~Pa} \mathrm{~s}$ \\
\hline 13 & Gasification temperature of THF & $T_{f}=339.15 \mathrm{~K}$ & 30 & Permeability of sediments & $k_{g}=10^{-15} \mathrm{~Pa} \mathrm{~s}$ \\
\hline 14 & Vaporization of water & $T_{w D}=373.15 \mathrm{~K}$ & 31 & Elastic module of sediments & $E=5 \times 10^{8} \mathrm{~Pa}$ \\
\hline 15 & Density of water & $\rho_{w}=1000 \mathrm{~kg} \mathrm{~m}^{-3}$ & 32 & Equilibrium pressure & $P_{e}=1 \times 10^{7} \mathrm{~Pa}$ \\
\hline 16 & Enthalpy of water vaporization & $\Delta H_{w-w g}=2.2 \times 10^{6} \mathrm{~J} \mathrm{~kg}^{-1}$ & 33 & Time step & $\Delta t=10^{-4}$ (dimensionless) \\
\hline 17 & Specific heat of water vapor & $C_{w g}=1385 \mathrm{~J} \mathrm{~kg}^{-1} \mathrm{~K}^{-1}$ & 34 & Space step & $\Delta h=10^{-2}$ (dimensionless) \\
\hline
\end{tabular}

a) Label 1-5 are designed by experiment; 6-10 refer to refs. [13,21]; 11-13 are obtained from http://cameochemicals.noaa.gov/chris/THF.pdf; 17-32 refer to refs. $[21,22]$. 
This work was supported by the National High Technology Research and Development Program of China (Grant N. 2006AA09A209-7), the Major Oil and Gas Program of China (Grant No. 2008ZX05026-04-011), Knowledge Innovative Program of the Chinese Academy of Sciences (Grant No. KJCX2-YW-LO2) and the National Natural Science Foundation of China (Grant No. 1077218), Meanwhile, Zhao Jing and Wang Ailan participated in the experimental process. We thank Prof. Li Yong for modifying the English writing.

1 Dickens G R. The potential volume of oceanic methane hydrates with variable external conditions. Org Geochem, 2001, 32: 1179-1193

2 Buffett B A, Archer D. Global inventory of methane clathrate: Sensitivity to changes in the deep ocean. Earth Planet Sci Lett, 2004, 227: 185-199

3 Kvenvolden K A. Potential effects of gas hydrate on human welfare. Proc Natl Acad Sci, 1999, 96: 3420-3426

4 Koh C A. Towards a fundamental understanding of natural gas hydrates. Chem Soc Rev, 2002, 31: 157-167

$5 \mathrm{Xu} \mathrm{W}$, Germanovich L N. Excess pore pressure resulting from methane hydrate dissociation in marine sediments: A theoretical approach. J Geophys Res, 2006, 111: B01104

6 Sultan N. Comment on "Excess pore pressure resulting from methane hydrate dissociation in marine sediments: A theoretical approach" by Wenyue Xu and Leonid N. Germanovich. J Geophys Res-Solid Earth, 2007, 112: NIL78-NIL84

7 Briaud J L, Chaouch A. Hydrate melting in soil around hot conductor. J Geotech Geoenvion Eng, 1997, 123: 645-653

8 Chaouch A, Briaud J L. Post melting behavior of gas hydrates in soft ocean sediments. In: Proceedings of the 29th Offshore Technology Conference. Texas: Offshore Technology Conference, 1997. 217-224

9 Sultan N, Cochonat P, Foucher J P, et al. Effect of gas hydrates melting on seafloor slope instability. Mar Geology, 2004, 213: 379-401

10 Chaouch A, Briaud J L. Hydrate Melting and Related Foundation Problems. Technical Report, Offshore Technology, Texas A\&M University, 1994
11 Nagashima K. Interferometric observation of mass transport processes adjacent to tetrahydrofuran clathrate hydrates under nonequilibrium conditions. Fluid Phase Equilibria, 2003, 214: 11-24

12 Tomoyuki IID A. Formation and dissociation of clathrate in stoichiometric tetrahydrofuran-water mixture subjected to one-dimensional cooling or heating. Chem Eng Sci, 2001, 56: 4747-4758

13 Akiya T, Shimazaki T, Oowa M. Formation characteristics of tetrahydrofuran hydrate to be used as a cool storage medium. In: Proceedings of 62nd Annual Meeting of The Society of Chemical Engineers. Tokyo: The Society of Chemical Engineers, 1997. 174

14 Ma Y H, Gou L T, He X X, et al. Experimental research of formation process of methane hydrate in freezing coarse grain size sand by computerized tomography (in Chinese). Nat Gas GeoSci, 2006, 17: 244-248

15 Xie Y M, Liang D Q, Guo K H, et al. Kinetics of Tetrahydrofuran hydrate formation decomposition outside of a heat transfer tube (in Chinese). J Xi' an Jiaotong Univ, 2005, 39: 313-316

16 Nagashima K, Orihashi S, Yamamoto Y, et al. Encapsulation of saline solution by tetrahydrofuran clathrate hydrates and inclusion migration by recrystallization. J Phys Chem B, 2005, 109: 10147-10153

17 Zeng H, Wilson L D, Walker V K, et al. The effect of biological and polymeric inhibitors on methane gas hydrate growth kinetics. J Am Chem Soc, 2006, 128: 2844-2850

18 Ullerich J W, Selim M S, Sloan E D. Theory and measurement of hydrate dissociation (in Chinese). AIChE J, 1987, 33: 747-752

19 Ahmadi G, Ji C, Smith H. Numerical solution for natural gas production from methane hydrate dissociation. J Pet Sci Eng, 2004, 41: 269-285

20 Zhang X H, Liu Y H, Li Q P, et al. Study on the dissociation scope of gas hydrate in deposit around heat conductor. Mech Eng, 2010, 32: 39-41

21 Wang S Y, Lu X B. Advances of research on mechanical properties of gas hydrate deposits (in Chinese). Adv Mech, 2009, 39: 176-188

22 Bai Y H, Li Q P, Yu X C, et al. Numerical study on dissociation of gas hydrate and its sensitivity to physical parameters. China Ocean Eng, 2007, 21: 625-636 